\title{
OSTRÁCODOS DE LAGOS SOMEROS DE LA REGIÓN CENTRAL DE ARGENTINA: IMPLICACIONES PALEOLIMNOLÓGICAS
}

\author{
ROMINA GISELA KIHN \\ Instituto de Ciencias de la Tierra y Ambientales de La Pampa, CONICET. Calle Mendoza, 109, Santa Rosa, 6300, \\ La Pampa, Argentina.rgkihn@gmail.com
}

FLORENCIA CRESPO

Facultad de Ciencias Exactas y Naturales, Universidad Nacional de La Pampa. Av. Uruguay 151, Santa Rosa 6300, La Pampa, Argentina. florr.crespo@gmail.com

\section{JOSÉ LUIS PALL}

Facultad de Ciencias Exactas y Naturales, Universidad Nacional de La Pampa, CONICET. Av. Uruguay 151, Santa Rosa, 6300, La Pampa, Argentina. pall.joseluis@gmail.com

\begin{abstract}
OSTRACODS OF SHALLOW LAKES OF THE CENTRAL REGION OF ARGENTINA: PALEOLIMNOLOGICAL IMPLICATIONS. Studies about ostracods in shallow lakes are very scarce in the Province of La Pampa, thus the objective of this work is to contribute to the knowledge of the species present in lentic environments, studying the distribution, abundance, and specific associations of ostracods. The samples come from four shallow lakes of the Pampean Region, Province of La Pampa, Argentina. Sampling was performed along transects perpendicular to the shore. Five genera represented by six species of benthic ostracods were identified. Heterocypris similis was present in all sampled water bodies, but was dominant in the Monte de Caldén (LCM) and Don Tomás (LDT) lagoons. Cypridopsis vidua was very abundant in the LCM. The species Chlamydotheca incisa and Potamocypris unicaudata were only recorded in the Ojo de Agua Lagoon (LCS). C. incisa is recorded for the first time in the province, and P. unicaudata in Argentina. The qualitative and quantitative variations of the ostracod assemblages can be related to topography, sedimentology, vegetation and salinity variations. The results of this study are important in the interpretation of the environments recorded during the Quaternary, since these species are also registered as fossils.
\end{abstract}

Key words: ostracods, lakes, La Pampa, modern analogues, Argentina.

RESUMO - Estudos sobre ostracodes em lagoas pouco profundas são muito escassos na Província de La Pampa, Argentina, e, portanto, o objetivo deste trabalho é contribuir para o conhecimento das espécies presentes nos ambientes lenticais, estudar distribuição, abundância e associações específicas de ostracodes. As amostras provêm de quatro lagoas da Região Pampeana, Província de La Pampa, Argentina. Amostragem sazonal foi realizada ao longo de um corte transversal perpendicular à costa, tendo sido identificado um total de cinco gêneros representados por seis espécies de ostracodes bentônicos. Heterocypris similis está presente em todos os corpos de água amostrados, mas é dominante nas lagunas Monte de Caldén (LCM) e Don Tomás (LDT). Cypridopsis vidua é muito abundante na LCM. As espécies Chlamydotheca incisa e Potamocypris unicaudata somente foram registradas na Laguna Ojo de Agua (LCS). C. incisa é o primeiro registro para a província e $P$. unicaudata é a primeira nomeação para a Argentina. As variações qualitativas e quantitativas dos conjuntos ostracodes podem estar relacionadas à topografia, sedimentologia, vegetação e variações de salinidade. Os resultados deste estudo são importantes na interpretação dos ambientes registrados durante o Quaternário, uma vez que estas espécies também ocorrem como fósseis.

Palavras-chave: ostracodes, lagoas, La Pampa, análogos modernos, Argentina.

\section{INTRODUCCIÓN}

Los ostrácodos son microcrustáceos bivalvos con caparazón quitino-calcáreo, que habitan todo tipo de medios acuáticos (desde continentales hasta marinos profundos, pasando por los estuarinos); en ambientes continentales su distribución está condicionada por las características físico-químicas del medio. Son muy sensibles a los cambios ecológicos que se relacionan no sólo con variaciones en las comunidades, sino también, con cambios morfológicos y estructurales del caparazón, a nivel de individuo. Estas características faunísticas permiten que los ostrácodos sean utilizados como indicadores de parámetros ambientales tanto bióticos como abíóticos. Es por ello que el conocimiento de la autoecología de las distintas especies posibilita conocer la evolución de los principales parámetros ambientales tanto en secuencias fósiles como actuales (Mezquita et al., 2005; Martín-Rubio et al., 2006). El estudio micropaleontológico de sedimentos recientes es una técnica muy utilizada, ya que proporciona datos directamente aplicables en las reconstrucciones paleoambientales (Martín-Rubio et al., 2006). Hasta el momento son relativamente escasos los estudios de ostrácodos actuales en la región Pampeana, pudiéndose citar a Ramírez (1967) y Laprida $(2006,2009)$, por 
lo que es muy importante la realización de nuevos muestreos y estudios.

En el presente trabajo se muestrearon cuatro cuerpos de agua en la Provincia de La Pampa con el objetivo de conocer la ocurrencia de las especies de ostrácodos en los distintos hábitats y relacionarla con características de estos ambientes (profundidad, vegetación, $\mathrm{pH}$, temperatura, salinidad, turbidez). Se pretende determinar qué especies viven en cada uno de los ambientes y qué condiciones ambientales condicionan su distribución. A partir de esta información se intenta contribuir con nuevos datos a la utilización de los ostrácodos en la interpretación de paleoambientes cuaternarios, particularmente en la región Pampeana.

\section{ÁREA DE ESTUDIO}

La Provincia de La Pampa está ubicada en la región central de Argentina e integra el dominio de los climas templados y semiáridos. Hacia el oeste y sudoeste, disminuye el nivel de precipitaciones y calidad de los suelos, siendo las amplitudes térmicas muy pronunciadas, típicas de los climas continentales. Las lluvias oscilan entre los $750 \mathrm{~mm}$ anuales en el ángulo noreste y los $150 \mathrm{~mm}$ en el extremo sudoeste. Las grandes variaciones de temperatura van de máximas en verano, con más de $40^{\circ} \mathrm{C}$, a mínimas en invierno, inferiores a $\operatorname{los} 7^{\circ} \mathrm{C}$ bajo cero. Los ecosistemas acuáticos de la provincia se encuentran sometidos a múltiples presiones, factores como sequía, eutrofización, aumento de la cantidad de materia orgánica, acidificación, turbidez o presencia de tóxicos, afectan a las comunidades de ostrácodos. En el caso de las lagunas someras, suelen estar colonizadas por macrófitos y no presentan estratificación térmica ya que su escasa profundidad $(<3 \mathrm{~m})$ genera una mezcla constante del agua (polimixis) (Scheffer, 2001a, b). La acción del viento facilita la interacción entre el agua y el sedimento y los nutrientes se reciclan rápidamente, aumentando así la productividad (Bécares et al., 2004).

\section{Características de los cuerpos de agua muestreados}

Laguna Monte de Caldén (LCM) $\left(37^{\circ} 22^{\prime} \mathrm{S}, 63^{\circ} 41^{\prime} \mathrm{O}\right)$. Se caracteriza por ser un cuerpo de agua de poca extensión (longitud máxima de $300 \mathrm{~m}$ y ancho de $100 \mathrm{~cm}$ ) con una profundidad máxima de $0,5 \mathrm{~m}$ aproximadamente,y aguas muy turbias con gran cantidad de materia orgánica en suspensión producto de la descomposición de restos de la vegetación circundante. Está rodeada por vegetación terrestre (Stipa brachychaeta). En las proximidades del cuerpo de agua se encuentra un monte de caldén (Prosopis caldenia) (Figuras 1, 2B).

Laguna Gral. Campos (LGC) $\left(37^{\circ} 29^{\prime} \mathrm{S}, 63^{\circ} 32^{\prime} \mathrm{O}\right)$. Laguna con una profundidad máxima de $3 \mathrm{~m}$ aproximadamente; la longitud máxima y el ancho son 1200 y 980 m respectivamente; el fondo es mayoritariamente rocoso, sus aguas poco turbias, y tiene vegetación en la costa (Figuras 1,2A).

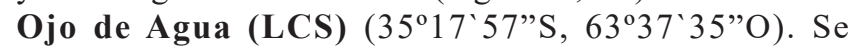
caracteriza por presentar una profundidad máxima promedio de $1,8 \mathrm{~m}$, una longitud máxima de $500 \mathrm{~m}$ y un ancho de 200 $\mathrm{cm}$. Recibe aporte de aguas subterráneas y de precipitación. Presenta abundante desarrollo de vegetación acuática del género Lemna, y períodos de marcada floración algal. Gran cantidad de juncáceas se encuentran rodeando el perímetro de la laguna y forman una isla en la zona central (Figuras 1,2C). Laguna Don Tomás (LDT) (36 $16^{\circ} 18.31^{\prime}$ 'S, 64¹8'49.02”O). Es un cuerpo de agua hipereutrófico superficial. Tiene una profundidad media de $2,3 \mathrm{~m}$, que varía según la estación, y cubre un área de 135,2 ha. La longitud máxima y el ancho son 1565 y $1181 \mathrm{~m}$ respectivamente. La laguna se caracteriza por su baja salinidad y transparencia (Echaniz et al., 2008) (Figuras 1, 2D).

Sin bien todos los cuerpos de agua muestreados son de carácter permanente, sufren retracciones y expansiones en su superficie debido a períodos de sequía y períodos lluviosos. Son cuerpos de agua someros sin estratificación térmica. Debido a su escasa profundidad sufren mezclas constantes por acción del viento.

\section{MATERIAL Y MÉTODOS}

El trabajo de campo consistió en colecta de muestras de agua y sedimento; en las lagunas LDT y LCM se realizó una transecta con cinco puntos de muestreo desde la orilla hacia el interior de la laguna, distanciados $20 \mathrm{~cm}$ unos de otros. En las lagunas LGC y LCS, donde la profundidad máxima se encuentra muy cercana a la costa, se tomaron cinco puntos de muestreo en una línea paralela a la línea de costa, con una distancia de $2 \mathrm{~m}$ entre muestras. Todo el muestreo se realizó durante el verano de 2016. Para la extracción de las muestras de sedimento se utilizó un aro metálico de $10 \mathrm{~cm}$ de diámetro por $2 \mathrm{~cm}$ de alto, y se recogieron los $2 \mathrm{~cm}$ superficiales. En campo se obtuvieron datos de profundidad máxima, transparencia empleando el disco de Secchi, temperatura y pH mediante una sonda multiparamétrica OAKTON DO6+. La salinidad ( $\mathrm{g} / \mathrm{L})$ se estimó multiplicando la conductividad $(\mathrm{mS} / \mathrm{cm})$ por el factor 0,67 (Echaniz, com. pers.).

Todas las muestras fueron desagregadas con $\mathrm{H}_{2} \mathrm{O}_{2}$ de 130 volúmenes diluida en cuatro partes de $\mathrm{H}_{2} \mathrm{O}$. Posteriormente, se tamizaron en húmedo con un tamiz de $63 \mu \mathrm{m}$ de luz de malla y se llevaron a estufa a $50^{\circ} \mathrm{C}$. Una vez secas, se estudiaron bajo lupa binocular; mediante la técnica de picking se extrajo el total de ejemplares presente. Para la determinación sistemática de los ostrácodos a nivel supragenérico se siguió a Moore \& Pitrat (1961). A nivel específico se utilizó bibliografía actualizada (Bertels \& Martínez, 1990; Cusminsky \& Whatley, 1996; Cusminsky et al., 2005; Ferrero, 2006; Laprida, 2006; Meisch, 2000; Karanovic, 2012). Para diferenciar los ejemplares vivos (biocenosis) de los ejemplares muertos (tanatocenosis) al momento del muestreo se observó la presencia de partes blandas y apéndices en los caparazones; no se utilizó la técnica de coloración con Rosa de Bengala, ya que esta técnica no es recomendada por algunos autores por teñir no sólo ejemplares vivos sino también ejemplares muertos con restos de materia orgánica (ver Danielopol et al., 2002 y referencias en él citadas). Ambos grupos (caparazones 


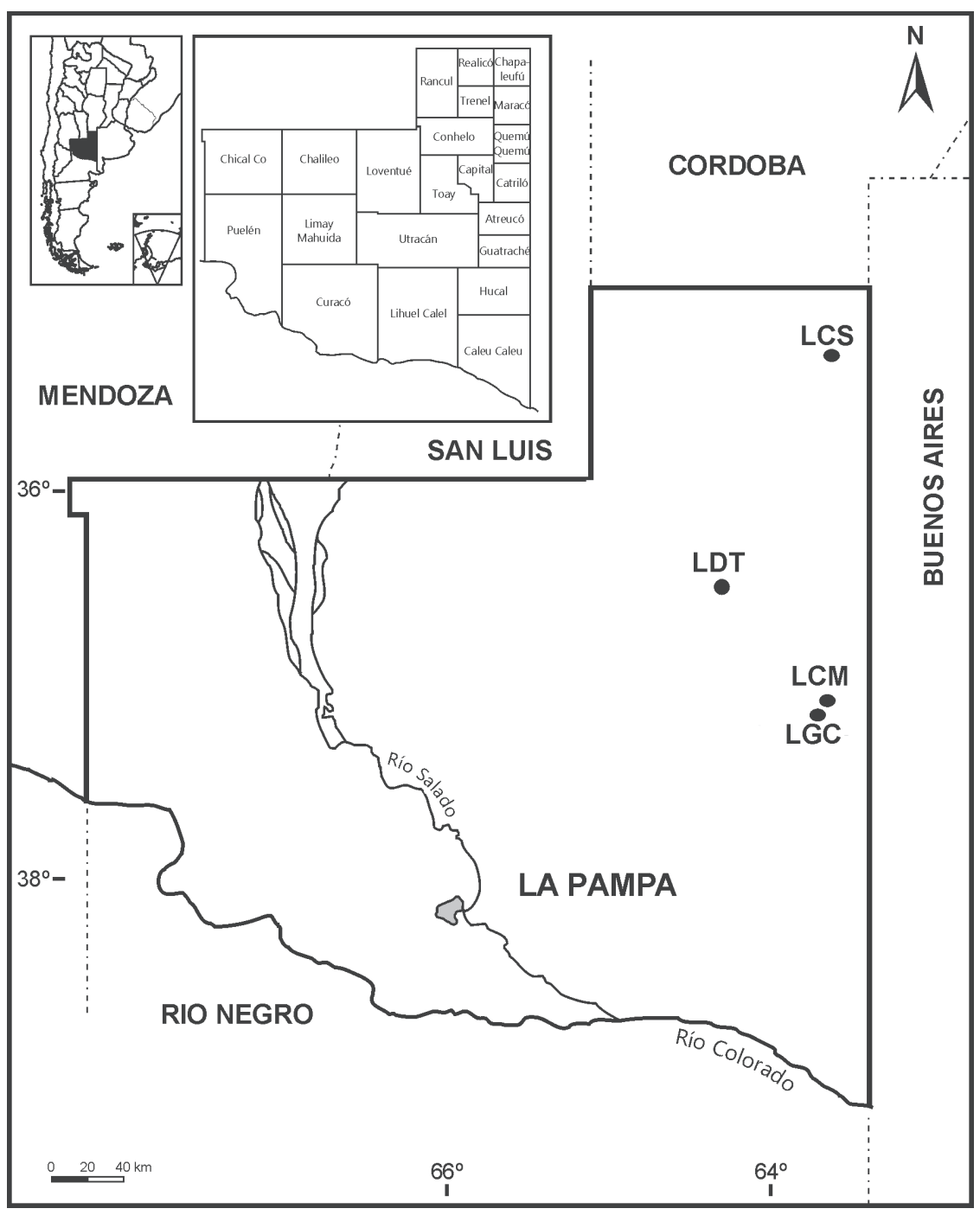

Figura 1. Localización geográfica de las lagunas muestreadas en la Provincia de La Pampa, Argentina. Abreviaturas: LGC, Laguna General Campos; LCM, Laguna Monte de Caldén); LDT, Laguna Don Tomás; LCS, Ojo de Agua.

Figure 1. Geographic location of lakes sampled in the Province of La Pampa, Argentina. Abbreviations: LGC, Laguna General Campos; LCM, Laguna Monte de Caldén); LDT, Laguna Don Tomás; LCS, Ojo de Agua.

con apéndices y caparazones vacíos) fueron utilizados para realizar estudios cualitativos y cuantitativos.

Todo el material estudiado en el presente trabajo se encuentra depositado en el Museo Provincial de Historia Natural (MHNLPam-ZE).

\section{Análisis de las poblaciones de ostrácodos}

Se contabilizó el total de ejemplares adultos y juveniles de todas las muestras estudiadas y se calculó la proporción adultos/juveniles. El hallazgo de poblaciones completas, es decir, con una proporción de adultos/juveniles igual a $1: 8(0,12)$, ocurre únicamente en ambientes con muy baja energía y tasa de sedimentación elevada, con sedimento de grano fino (limo-arcillas) (Whatley, 1988a,b). Si se considera la dinámica del ambiente estudiado, las tanatocenosis están sujetas a desplazamientos; sin embargo, la presencia de poblaciones con valvas adultas y determinada proporción de valvas juveniles permite establecer que, si bien pudieron haber sido removidas en algún grado, no han sido desplazadas de sus hábitats originales.

Con el fin de determinar el grado de estabilidad ambiental, se calculó el índice de diversidad de Shannon-Wiener, el cual es considerado un índice de heterogeneidad que incluye tanto el número de especies como la distribución de individuos entre especies. Este valor se obtiene al aplicar la función de información $\mathrm{H}$ incluida en la siguiente ecuación:

$$
\mathrm{H}(\mathrm{S})=-\Sigma \text { pi. } \ln \mathrm{pi}
$$

Siendo $\mathrm{S}$ el número de especies en cada muestra y pi la proporción de individuos de la especie i, es decir, $\mathrm{p}=\mathrm{ni} / \mathrm{N}$, donde ni es el número de individuos de la especie i y $\mathrm{N}$ es la abundancia total de las especies (Murray, 1973). A partir del estudio de la diversidad de los foraminíferos en ambientes marinos se determina que los índices mayores a $2,5 \mathrm{se}$ relacionan con ambientes marinos normales, mientras que los 

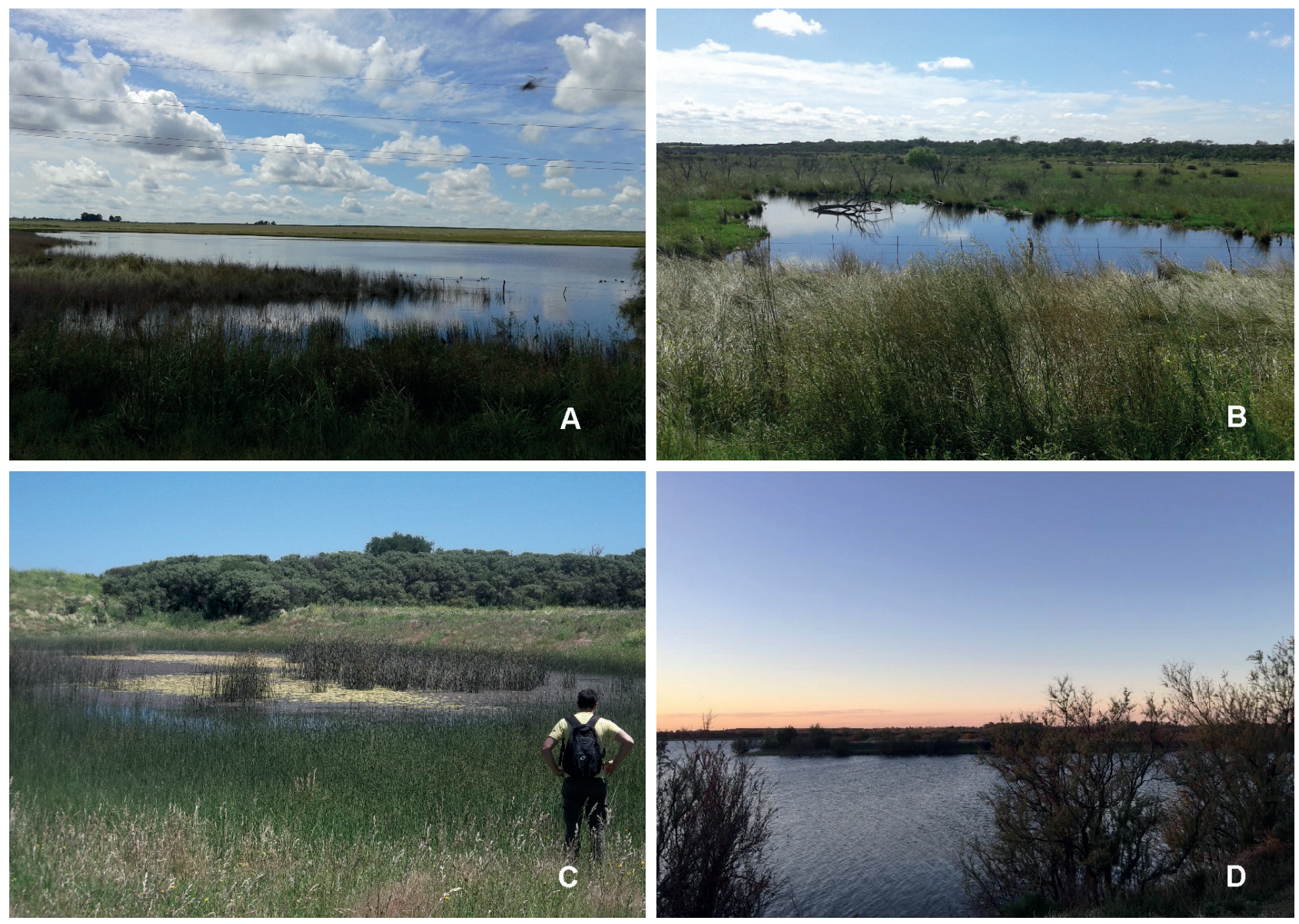

Figura 2. Vistas generales de los cuerpos de agua estudiados. A, Laguna Gral. Campos (LGC); B, Laguna Monte de Caldén (LMC); C, Ojo de Agua (LCS); D, Laguna Don Tomás (LDT).

Figure 2. General views of the bodies of water studied. A, Laguna Gral. Campos (LGC); B, Laguna Monte de Caldén (LMC); C, Ojo de Agua (LCS); D, Laguna Don Tomás (LDT).

valores menores indican ambientes marinos más inestables o restringidos, en los cuales los taxones son escasos o bien existen especies dominantes (Buzas \& Gibson, 1969; Gibson \& Buzas, 1973). Este índice ha sido aplicado con anterioridad en asociaciones de ostrácodos obteniendo resultados que aportaron importante información sobre el ambiente y la estabilidad ambiental (Martínez, 2002, 2005; Gómez et al., 2005; Ferrero, 2006, 2009; Kihn et al., 2016, 2017).

\section{RESULTADOS}

$\mathrm{El} \mathrm{pH}$ varió entre 7,5 y 8,2 y la temperatura del agua fue de entre $17-20^{\circ} \mathrm{C}$. La salinidad en los lagos LCS, LCM y LDT fue baja $(1,42-3,07 \mathrm{~g} / \mathrm{L})$, siendo menor en LCS; el cuerpo de agua LGC fue la que presentó mayor salinidad (18,08 g/L). Las lagunas LDT y LCM presentaron aguas con una alta turbidez (15 y $5 \mathrm{~cm}$ respectivamente) (Tabla 1$)$.

Se determinó un total de seis especies pertenecientes a cinco géneros y dos familias de ostrácodos bentónicos. Las lagunas LDT y LCM fueron las que presentaron las mayores densidades 786 y 618 individuos/ 25 gr de sedimento; mientras que los cuerpos de agua LCS y LGC se caracterizaron por presentar baja densidad de ostrácodos con valores de 66 y
Tabla 1. pH, temperatura, salinidad y transparencia (disco de Secchi) de las lagunas muestreadas. Abreviaturas: LGC, Laguna General Campos; LCM, Laguna Monte de Caldén); LDT, Laguna Don Tomás; LCS, Ojo de Agua.

Tabla 1. $\mathrm{pH}$, temperature, salinity and transparency (Secchi disk) of the sampled lakes. Abbreviations: LGC, Laguna General Campos; LCM, Laguna Monte de Caldén); LDT, Laguna Don Tomás; LCS, Ojo de Agua.

\begin{tabular}{ccccc}
\hline Laguna & $\mathrm{pH}$ & $\mathrm{T}^{\circ} \mathrm{C}$ & $\begin{array}{c}\text { Salinidad } \\
(\mathrm{g} / \mathrm{L})\end{array}$ & $\begin{array}{c}\text { Transparencia } \\
(\text { disco Sechi) }\end{array}$ \\
\hline LGC & 8,2 & 17 & 18,08 & $260 \mathrm{~cm}$ \\
LCM & 7,8 & 20 & 3,07 & $5 \mathrm{~cm}$ \\
LDT & 7,5 & 18 & 2,05 & $15 \mathrm{~cm}$ \\
LCS & 7,9 & 18 & 1,42 & $160 \mathrm{~cm}$ \\
\hline
\end{tabular}

13 individuos/25 gr de sedimento. El índice de ShannonWiener presentó valores que variaron entre 2 y 0,5 . De las seis especies presentes en este estudio, Chlamydotheca incisa y Potamocypris sp. solo se encontraron en la laguna LCS, Heterocypris similis y H. incongruens fueron muy abundantes en los cuerpos de agua LDT y LCM, Limnocythere cusminskyae presentó poblaciones completas y muy desarrolladas en la laguna LDT y Cypridopsis vidua estuvo presente en las lagunas LDT; LCS y LCM (Tabla 2). 
Tabla 2. Número de individuos (indiv./25 g de sedimento) de las especies por sitio muestreado. Abreviaturas: LGC, Laguna General Campos; LCM, Laguna Monte de Caldén); LDT, Laguna Don Tomás; LCS, Ojo de Agua; V, vivos; T, total.

Table 2. Number of individuals (indiv./25 g of sediment) of each species per sampled site. Abbreviations: LGC, Laguna General Campos; LCM, Laguna Monte de Caldén); LDT, Laguna Don Tomás; LCS, Ojo de Agua; V, living; T, total.

\begin{tabular}{|c|c|c|c|c|c|c|c|c|}
\hline \multirow[t]{3}{*}{ Especies de ostrácodos } & \multicolumn{8}{|c|}{ Lagunas muestreadas } \\
\hline & \multicolumn{2}{|c|}{ LGC } & \multicolumn{2}{|c|}{ LCM } & \multicolumn{2}{|c|}{ LDT } & \multicolumn{2}{|c|}{ LCS } \\
\hline & $\mathrm{V}$ & $\mathrm{T}$ & $\mathrm{V}$ & $\mathrm{T}$ & $\mathrm{V}$ & $\mathrm{T}$ & $\mathrm{V}$ & $\mathrm{T}$ \\
\hline Chlamydotheca incisa & 0 & 0 & 0 & 0 & 0 & 0 & 1 & 8 \\
\hline Heterocypris similis & 1 & 4 & 70 & 291 & 56 & 350 & 2 & 14 \\
\hline Heterocypris incongruens & 0 & 0 & 24 & 93 & 47 & 150 & 4 & 30 \\
\hline Potamocypris unicaudata & 0 & 0 & 0 & 0 & 0 & 0 & 0 & 9 \\
\hline Cypridopsis vidua & 0 & 0 & 25 & 219 & 15 & 25 & 0 & 5 \\
\hline Limnocythere cusminskyae & 1 & 5 & 1 & 8 & 20 & 216 & 0 & 0 \\
\hline
\end{tabular}

\section{SISTEMÁTICA}

Phylum CRUSTACEA Pennant, 1777

Clase OSTRACODA Latreille, 1806

Orden PODOCOPIDA G. W. Müller, 1894

Suborden PODOCOPA Sars, 1866

Superfamilia CYPRIDOIDEA Baird, 1845

Familia CYPRIDIDAE Baird, 1845

Chlamydotheca Saussure, 1858

Especie tipo. Cypris (Chlamydotheca) azteca Saussure, 1858.

Chlamydotheca incisa (Claus, 1893)

(Figura 3A)

1893 Pachycypris incisa Claus, 205; lám. 8, figs. 7-15.

Distribución geográfica. Extensamente distribuida por América del Sur, habiéndose registrado en Argentina, Bolivia, Brasil, Chile, Paraguay, Perú y Uruguay (Martens \& Behen, 1994). También fue hallada en sedimentos cuaternarios (Bertels \& Martínez, 1990; Martínez, 2002) y actuales (Ramírez, 1967; César et al., 2004) de la provincia de Buenos Aires.

\section{Heterocypris Claus, 1893}

Especie tipo. Cypris incongruens Rahmdor, 1808.

Heterocypris similis (Wierzejski en Ramírez, 1967)

(Figuras 3B-C)

1967 Cyprinotus similis Wierzejski. Ramírez, p. 29-30; lám. 5, figs. 27-32.

Distribución geográfica. Se encuentra en ambientes no marinos de Argentina y Brasil (Martens \& Behen, 1994). Según Moguilevsky \& Whatley (1995), las especies de este género habitan charcas temporarias y lagunas permanentes. Laprida (2006) encuentra ejemplares de esta especie en cuerpos de agua de carácter permanente en la provincia de Buenos Aires.
Heterocypris incongruens (Ramdohr, 1808)

(Figura 3D)

1808 Cypris incongruens Rahmdor, p. 86; lám. 3, figs. 1-12, 15-16 y 19-20.

Distribución geográfica. Es una especie de distribución cosmopolita; en América del Sur se la registró en Argentina, Brasil, Uruguay y Venezuela (Kotzian, 1974; Purper \& Würdig-Maciel, 1974; Martens \& Behen, 1994). Es frecuente en sedimentos no marinos del Cuaternario de la provincia de Buenos Aires (Ferrero, 1996) y en ambientes actuales del norte de la Patagonia (Schwalb et al., 2002) y provincia de Buenos Aires (Laprida, 2006).

\section{Cypridopsis Brady, 1867}

Especie tipo. Cypris vidua O.F. Müller, 1776.

Cypridopsis vidua (O.F. Müller, 1776)

(Figuras 3E-F)

1776 Cypris vidua O.F. Müller, p. 199.

Distribución geográfica. Especie de distribución cosmopolita; en Sudamérica se la encuentra en ambientes actuales de Argentina, Brasil, Paraguay, Uruguay y Venezuela (Martens \& Behen, 1994; César et al., 2004); en Argentina fue recuperada de localidades cuaternarias desde el Chaco hasta la Patagonia (Zabert, 1981; Zabert \& Herbst, 1986; Cusminsky, 1995; Laprida, 2006), en sedimentos holocenos del curso medio y desembocadura del Arroyo Napostá Grande, Bahía Blanca, Buenos Aires (Martínez, 2002) y en sedimentos actuales del estuario de Bahía Blanca (Martínez, 2005).

Potamocypris Brady, 1870

Potamocypris unicaudata Schäfer, 1943

(Figura 3G)

1943 Potamocypris unicaudata Schäfer, p. 210-216.

Distribución geográfica. Es de distribución cosmopolita, 
se lo ha encontrado en diversos cuerpos de agua dulce o de baja salinidad; es la primera cita para Argentina, si bien los ejemplares de Laprida (2006) podrían asignarse a esta especie.

Familia LIMNOCYTHERIDAE Klie, 1938

\section{Limnocythere Brady, 1868}

Especie tipo. Cythere inopinata Baird, 1843.

Limnocythere cusminskyae Ramón-Mercau, Plastani \& Laprida, 2014

(Figuras 3H-J)

2014 Limnocythere cusminskyae Ramón-Mercau, Plastani \& Laprida, p. 26-36.figs. 2(a-g) y 3(a-h).

Distribución geográfica. La especie ha sido registrada tanto viva como fósil en la Pampa sudoriental, principalmente en ubicaciones cerca de la costa atlántica. Laprida (2006) la encontró Chascomús, Salada Grande, Los Horcones, Laguna del Monte y en el Arroyo Grande. Bertels \& Martínez (1990, 1997) registraron la especie en sedimentos del Holoceno de una sección expuesta a lo largo del margen del Napostá Grande. Ferrero (1996) la halló en un afloramiento del Holoceno del Río Quequén Grande y en sedimentos del Pleistoceno Tardío.

Análisis de la estructura de las poblaciones. En las muestras estudiadas la mayoría de las especies presentaron proporciones de valvas de adultos/juveniles (VA/ VJ) comprendidas entre $1 / 6(0,16)$ y $1 / 3(0,33)$, que reflejan condiciones ambientales de baja energía (Brouwers, 1988; Coimbra et al., 2007). Estas poblaciones están constituidas por sus formas adultas y la mayoría de los estados juveniles o mudas que indican un desplazamiento mínimo (poblaciones del tipo A, Whatley, 1988b) (Tabla 3). Se encontraron ejemplares vivos de Cypridopsis vidua, Heterocypris similis, H. incongruens y Limnocythere cusminskyae; Chlamydotheca incisa. De esta última sólo se recuperó un ejemplar adulto vivo en LCS (Tabla 1). Potamocypris sp. fue la única especie de la que no se encontraron ejemplares vivos; sin embargo, las valvas y caparazones recuperados no presentaban evidencias de transporte ni de largos períodos de exposición.

La estructura poblacional post mortem de los ostrácodos está determinada no sólo por los factores ambientales y la dinámica del ambiente depositacional, sino también por el tamaño y la morfología de los caparazones de cada especie, ya que especies con valvas muy ornamentadas y charnelas complejas tienen menor probabilidad de ser desarticuladas y fragmentadas después de su muerte (Kontrovitz, 1975). En las muestras estudiadas en este trabajo se observó que las especies de menor tamaño y formas globosas como Cypridopsis vidua se preservan mejor que aquellas con caparazones más grandes y poco calcificados como Chlamydotheca incisa, los que son más susceptibles a la fragmentación. Numerosos fragmentos asignables a esta especie fueron recuperados en el cuerpo de agua LCS.
Distribución cualitativa de las asociaciones de ostrácodos autóctonos. Se analizó la composición de las biocenosis y tanatocenosis, teniendo en cuenta las condiciones hidrológicas, sedimentológicas y la presencia o ausencia de vegetación, en los distintos hábitats o subambientes del área estudiada. Todos los taxones de ostrácodos de las biocenosis y tanatocenosis autóctonas analizadas en este estudio pertenecen a la superfamilia Cytheroidea, y son típicamente bentónicos. Si se considera que se han tomado $2 \mathrm{~cm}$ de sedimento superficial, dentro de las tanatocenosis se incluyen valvas que por procesos tafonómicos representan un time-averaging de generaciones (Kidwell \& Bosence, 1991). Las tanatocenosis autóctonas halladas en los lagos estudiados están constituidas por una mezcla de poblaciones no contemporáneas que provienen de un mismo hábitat. Posiblemente las diferentes especies que integran las tanatocenosis autóctonas en este estudio reflejen cambios estacionales de salinidad, temperatura, oxígeno, etcétera; ya que los lagos estudiados sufren grandes retracciones en los períodos de sequía.

Por consiguiente, se considera que estas asociaciones de ostrácodos, por el efecto time-averaging, presentan un enriquecimiento en la composición faunística, que se relaciona con cortas fluctuaciones ambientales y/o cambios esporádicos no determinables (Staff et al., 1986), mientras que las biocenosis constituyen una generación de ostrácodos $\mathrm{y}$, consecuentemente, no reflejan variaciones ambientales (Martínez, 2005).

\section{DISCUSIÓN}

La región Pampeana presenta una gran diversidad de cuerpos de agua lénticos que son permanentes o temporarios, someros y de carácter eutrófico; carecen de estratificación química o térmica (Dangavs, 1998). En estos ambientes, la salinidad, la composición iónica y la estabilidad determinan los patrones de distribución de las poblaciones de ostrácodos. La Provincia de La Pampa posee condiciones de aridez más acentuadas que la Provincia de Buenos Aires (Cano, 1980) lo que conlleva a que la mayoría de los sistemas lagunares posean mayores fluctuaciones de nivel de agua y salinidad que las lagunas bonaerenses.

La baja diversidad registrada en el presente estudio (seis especies) y los bajos valores del índice de Shannon-Wiener se deberían a la inestabilidad ambiental, ya que estos lagos si bien son permanentes, sufren grandes retracciones y cambios en los períodos de sequía teniendo un comportamiento semipermanente y siendo ambientes de alta inestabilidad. La menor densidad registrada en la laguna LGC podría deberse a la mayor salinidad registrada en este sitio $(18,08 \mathrm{~g} / \mathrm{L})$. Por otro lado las mayores densidades registradas en las lagunas LDT y LCM podrían deberse a un enriquecimiento del sistema por aporte de nutrientes $(\mathrm{N}$ y $\mathrm{P})$ y materia orgánica producto de la entrada de aguas residuales de la localidad de Santa Rosa a la laguna LDT y por actividad ganadera en el cuerpo de agua LCM. Esto coincide con lo propuesto por Ruiz et al. (2013), quienes postulan que ante un aumento en estos nutrientes algunas especies se ven favorecidas y se produce un gran aumento en sus poblaciones. 


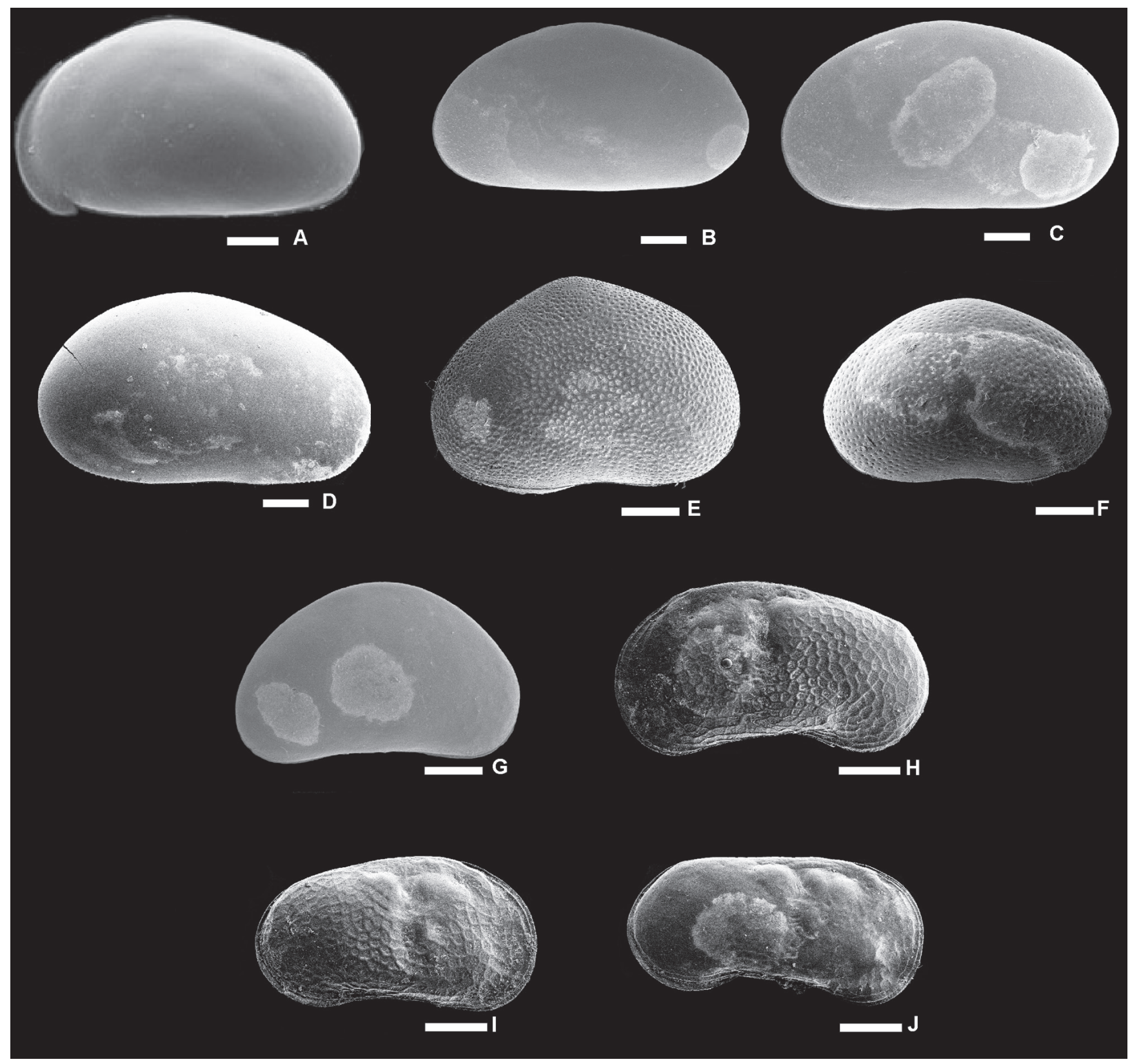

Figura 3/Figure 3. Biocenosis y tanatocenosis autóctonas/ autochthonous biocoenosis and thanatocoenosis. A, Chlamydotheca incisa, VI/ LV (MHNLPam-ZE 1); B, Heterocypris similis, VI/LV (MHNLPam-ZE 2); C, Heterocypris similis, VI/LV (MHNLPam-ZE 3); D, Heterocypris incongruens, VD/RV (MHNLPam-ZE 4); E, Cypridopsis vidua, VI/LV (MHNLPam-ZE 5); F, Cypridopsis vidua, VD/LV (MHNLPam-ZE 6); G, Potamocypris unicaudata, VD/RV (MHNLPam-ZE 7); H, Limnocythere cusminskyae, hembra/female VI/LV (MHNLPam-ZE 8); I, Limnocythere cusminskyae, hembra/female VD/RV (MHNLPam-ZE 9); J, Limnocythere cusminskyae, macho/male VD/RV (MHNLPam-ZE 10). Abreviaciones/ Abbreviations: VD/RV, valva derecha/right valve; VI/LV, valva izquierda/left valve. Escalas/Scale bars $=100 \mu \mathrm{m}$.

Laprida (2006) encuentra ejemplares de Chlamydotheca incisa solo en ambientes temporarios de escasa profundidad y vegetados; en el presente trabajo se encontró un ejemplar vivo de esta especie en un cuerpo de agua permanente de una profundidad máxima de $1,8 \mathrm{~m}$ con abundante vegetación de juncáceas (LCS), por lo que resultaría de interés realizar nuevos muestreos en otros cuerpos de agua someros permanentes. Por el contrario, en la laguna LCM, que es de menor profundidad y también presenta vegetación, con un índice de turbidez mayor que el de LCS, no se encontraron ejemplares de $C$. incisa. La ausencia de
C. incisa en el cuerpo de agua LCM podría indicar que la turbidez sería un factor condicionante en la distribución de esta especie, coincidiendo con lo establecido por Bronshtein (1947) y Argollo \& Mourguiart (2000), quienes dicen que la presencia de vegetación y la turbidez del agua son factores condicionantes en la distribución de las asociaciones de ostrácodos de ambientes continentales. El bajo número de C. incisa registrado podría atribuirse a la técnica utilizada para el muestreo por lo que sería recomendable en futuros muestreos utilizar además la técnica de recolección con red. 
Tabla 3. Número de ejemplares adultos y juveniles .(indiv./25g de sedimento). Abreviaturas: LGC, Laguna General Campos; LCM, Laguna Monte de Caldén); LDT, Laguna Don Tomás; LCS, Ojo de Agua.

Table 3. Number of adults and juveniles specimens.(indiv./25 g of sediment). Abbreviations: LGC, Laguna General Campos; LCM, Laguna Monte de Caldén); LDT, Laguna Don Tomás; LCS, Ojo de Agua.

\begin{tabular}{lcccccccc}
\hline \multicolumn{1}{c}{ Especies } & \multicolumn{2}{c}{ LGC } & \multicolumn{2}{c}{ LCM } & \multicolumn{2}{c}{ LDT } & LCS \\
\hline & A & J & A & J & A & J & J \\
Chlamydotheca incisa & 0 & 0 & 0 & 0 & 0 & 0 & 2 & 6 \\
Heterocypris similis & 0 & 4 & 50 & 241 & 52 & 298 & 2 & 12 \\
Heterocypris incongruens & 0 & 0 & 14 & 79 & 25 & 125 & 4 & 26 \\
Potamocypris unicaudata & 0 & 0 & 0 & 0 & 0 & 0 & 0 & 9 \\
Cypridopsis vidua & 0 & 0 & 30 & 189 & 2 & 23 & 0 \\
Limnocythere cusminskyae & 1 & 4 & 1 & 7 & 26 & 192 & 0 \\
\hline
\end{tabular}

Cypridopsis vidua fue abundante en el cuerpo de agua LCM y no se registró en la laguna LGC, mientras que en las lagunas LDT y LCS fue escasa, en contraposición a lo registrado por Laprida (2006) quien encuentra esta especie en forma abundante en todos los cuerpos de agua muestreados en la provincia de Buenos Aires. La laguna LCM presenta escasa profundidad $(50 \mathrm{~cm})$; esta característica hace que durante los períodos de sequía sufra grandes retracciones, lo cual lo vuelve un sitio cambiante e inestable y por lo tanto adverso para el desarrollo de algunas especies y favorece el mayor desarrollo de especies tolerantes a condiciones inestables y con alta turbidez (5 cm). D'Ambrosio (2014) halla C. vidua en toda la Cuenca de la Laguna de Llancanelo, pero con mayor abundancia en el delta del río Malargüe y en los arroyos; que en la laguna y los bañados. Kihn et al. (2017b) encuentran esta especie en todos los ambientes estudiados del Arroyo Punta del Agua; se la ha registrado em ambientes oligosalinos, por lo que la salinidad sería un factor limitante en su distribución.

Según Schwalb (2002) Heterocypris incongruens es una especie típica de ambientes temporales y su ciclo de vida corto es considerado una adaptación favorable para desarrollarse en ambientes inestables (Ganning, 1971), además es considerada una especie oportunista omnívora (Reichholf, 1983). Como se mencionó con anterioridad, los lagos estudiados en el presente trabajo son ambientes muy inestables debido a sus retracciones y expansiones; esto explicaría la presencia y abundancia de $H$. incongruens en todos los lagos analizados ya que es una especie que se adapta rápidamente a variaciones en el ambiente. D'Ambrosio (2014) encuentra esta especie en bañados, bañados termales y arroyos de la Laguna de Llancanelo; además la halla en el delta del Río Malargüe de la Provincia de Mendoza a pHs que fluctúan entre 7,6-9,2 y salinidades de entre 0,2 a $2,7 \mathrm{~g} / \mathrm{L}$; siendo más abundante en los bañados. Laprida (2006) la encuentra en ambientes costeros de lagunas oligohalinas de fuerte alcalinidad; $\mathrm{pH}=$ 8-12,8. Kihn et al. (2017) la citan para ambientes lénticos someros y lóticos de baja energía del arroyo Punta del Agua con $\mathrm{pH}=8,45-9,05$ y salinidades de $0,335-0,6365 \mathrm{~g} / \mathrm{L}$. Estos estudios indican que esta especie es altamente tolerante a $\mathrm{pHs}$ fuertemente alcalinos y aguas oligohalinas ya que en el presente estudio no se la observó en la laguna LGC que presentó una salinidad de $18 \mathrm{~g} / \mathrm{L}$.

Heterocypris similis fue hallada por Kihn et al. (2017b) en ambientes lénticos permanentes y semipermanentes someros, y ambientes lóticos de baja energía con $\mathrm{pH}=8,45-9,05$ y salinidades de 0,335-0,6365 g/L. Laprida (2006) la encuentra en lagunas permanentes, en ambientes lóticos y en charcas temporarias hipohalina- mesopoikilohalina positiva a oligohalina y $\mathrm{pH}=7,5-10,01$. D'Ambrosio (2014) la registra en un testigo de la Laguna Llancanelo. Esta especie podría ser considerada como un indicador de aguas eutróficas ya que se observó un marcado aumento en aquellos cuerpos de agua con mayor aporte de nutrientes como LDT, coincidiendo con lo planteado por Laprida (2006). Además Potamocypris unicaudata se registró en un bajo porcentaje en la laguna LCS, que es la que presentó menor salinidad $(1,42 \mathrm{~g} / \mathrm{L})$. Para esta especie la salinidad sería el principal factor condicionante para su distribución. Laprida (2006) encuentra ejemplares asignadas a Potamocypris sp. que son comparables con P. unicaudata en lagunas oligohalinas de la Provincia de Buenos Aires.

En la laguna LGC tanto la composición del sustrato (de tosca) serían características desfavorables para el desarrollo de ostrácodos bentónicos continentales. Los otros lagos estudiados presentaron fondos fangosos-arenosos.

\section{CONCLUSIÓN}

En las lagunas estudiadas se pudieron identificar seis especies pertenecientes a cinco géneros y a dos familias de ostrácodos bentónicos. En cuerpos límnicos de escasa profundidad estudiados en este trabajo la turbidez del agua, la presencia de vegetación y la composición del sustrato son factores condicionantes para el desarrollo de la ostracofauna. Chlamydotheca incisa y Potamocypris unicaudata se encontraron solo en el ojo de agua LCS, que presenta vegetación acuática y aguas poco turbias, por lo que podemos concluir que la presencia de vegetación y el grado de turbidez serían factores importantes en la distribución de estas especies. Se hallaron numerosos ejemplares de Cypridopsis vidua en cuerpos de agua con una elevada turbidez producto de un nivel elevado de eutrofización como Laguna Don Tomás (LDT) y Laguna Monte de Caldén (LCM). La mayor concentración de nutrientes y elevada turbidez del agua favorecería el desarrollo de esta especie. Hetrocypris similis y $H$. incongruens estuvieron presente en todos los lagos muestreados. El viento en estos cuerpos de agua sería un agente tafonómico importante, generando destrucción de un alto porcentaje de las valvas, por lo que la preservación de 
las valvas se ve muy limitada a las características propias de las especies, siendo más probable que queden en el registro aquellas con caparazones más robustos.

\section{AGRADECIMIENTOS}

Los autores de este trabajo queremos agradecer la lectura crítica, las observaciones y contribuciones de A. Vignatti y S. Echaniz que han mejorado el manuscrito; a los revisores L. Ferrero y anónimo quienes han enriquecido sustancialmente este trabajo. Este estudio fue financiado por el proyecto BID PICT 2014-1271, Beca CONICET posdoctoral. Los resultados son parte de la tesis de grado de F. Crespo.

\section{REFERENCIAS}

Argollo, J. \& Mourguiart, P. 2000. Late Quaternary climate history of the Bolivian Altiplano. Quaternary International, 72:37-51. doi:10.1016/S1040-6182(00)00019-7

Baird, W. 1845. Arrangement of the British Entomostraca, with a list of species, particularly noticing those which have as yet been discovered within the bounds of the club. Transactions of the Berkwickshire Naturalist' Club, 2:145-158.

Bécares E.; Conty, A.; Rodriguez-Villafañe, C. \& Blanco, S. 2004. Funcionamiento de los lagos someros mediterráneos. Ecosistemas, 13:2-12.

Bertels, A. \& Martínez, D.E. 1990. Quaternary ostracodes of continental and transitional littoral-shallow marine environments. Courier Forschchungs Institut Senckenberg, 123:141-159.

Bertels, A. \& Martínez, D. 1997. Ostrácodos holocenos de la desembocadura del Arroyo Napostá Grande, sur de la provincia de Buenos Aires, Argentina. Revista Española de Micropaleontología, 29:29-69.

Brady, G.S. 1867. A synopsis of the recent British Ostracoda. The Intellectual Observer, 12:110-130.

Brady, G.S. 1868. A monograph of the recent British Ostracoda. Transactions of the Linnean Society of London, 26:353-495. doi:10.1111/j.1096-3642.1968.tb00199.x

Bronshtein, Z.S. 1947. Fresh-water Ostracoda. In: Fauna of the USSR: Crustacean, Moscow, Academy of Sciences of the USSR Publishers, 445 p. (Russian Translation Series 64).

Brouwers, E.M. 1988. Sediment transportation detected from analysis of ostracod population structure: an example from the Alaskan continental shelf. In: P. Decker; J.P. Colin \& J.P. Peypouquet (eds.) Ostracoda in the Earth Sciences, Elsevier, p. 231-244.

Buzas, M.A. \& Gibson, T.G. 1969. Species diversity: benthonic foraminifera in western North Atlantic. Science, 163:72-75. doi:10.1126/science.163.3862.72

Cano, E. 1980. Inventario Integrado de los Recursos Naturales de la provincia de La Pampa. Buenos Aires, Instituto Nacional de Tecnología Agropecuaria (INTA), 493 p.

César, I.I.; Armendáriz, L.C. \& Damborenea, M.C. 2004. Ostrácodos (Crustacea) de la Isla Martín García, Río de la Plata, Argentina. Natura Neotropicalis, 32:147-151. doi:10.14409/natura. $v 2 i 32.3780$

Claus, C. 1893. Beitrage zur kenntniss der süsswasser- Ostracoden. I. Über den Korper und Gliedmassenbau der Cypriden, nebst Bemerkungen über einzelne organe derselben. Arbeiten aus Zoologische Institut der Universitat Wien zoologische, 10:147-216.

Coimbra, J.C.; Carreño, A.L.; Geraque, E.A. \& Eichler, B.B. 2007. Ostracodes (Crustacea) from Cananéia-Iguape estuarine/ lake system and geographical distribution of the mixohaline assemblages in southern and southeastern Brazil. Iheringia, Série Zoologia, 97:273-279. doi:10.1590/S0073-47212007000300010

Cusminsky, G.C. 1995. Ostrácodos lacustres cuaternarios en la transecta Tronador-Laguna Cari-Laufquen, Patagonia Norte, Argentina. In: CONGRESO ARGENTINO DE PALEONTOLOGÍA Y BIOESTRATIGRAFÍA, 6, 1994. Actas, Trelew, APA, p. 99-105.

Cusminsky, G.; Pérez, P.A.; Schwalb, A. \& Whatley, R. 2005. Recent lacustrine ostracods from Patagonia, Argentina. Revista Española de Micropaleontología, 37:431-450.

Cusminsky, G.C. \& Whatley, R. 1996. Quaternary non-marine ostracodes from lake beds in northern Patagonia. Revista Española de Paleontología, 11:143-154.

D’Ambrosio, S. 2014. Reconstrucción paleolimnológica de la Laguna Llancanelo (Mendoza, Argentina) a través del estudio de ostrácodos del Cuaternario. Facultad de Ciencias Naturales y Museo, Universidad Nacional de La Plata, Tesis Doctoral, 181 p.

Dangavs, N. 1998. Los ambientes lénticos de la pampasia bonaerense. In: A.F. Cirelli (ed.) Agua problemática regional, Eudeba, p. 145-152.

Danielopol, D.L.; Ito, E.; Wansard, G.; Kamiya, T.; Cronin, T.M. \& Baltanás, A. 2002. Techniques for collection and study of ostracoda. In: J.A. Holmes \& A. Chivas (eds.) The Ostracoda: applications in Quaternary research, Washington, American Geophysical Union, p. 65-97 (Geophysical Monograph Series 131). doi:10.1029/131GM04

Echaniz, S.A.; Vignatti, A.M. \& Bunino, P.C. 2008. El zooplancton de un lago somero hipereutrófico de la región central de Argentina: cambios después de una década. Biota Neotropica, 8:63-71. doi:10.1590/S1676-06032008000400005

Ferrero, L. 1996. Paleoecología de ostrácodos holocenos del estuario del río Quequén Grande (provincia de Buenos Aires). Ameghiniana, 32:209-222.

Ferrero, L. 2006. Micropaleontología y paleoecología del Cuaternario del sudeste de la provincia de Buenos Aires. Facultad de Ciencias Exactas y Naturales, Universidad Nacional de Mar del Plata, Tesis doctoral, 373 p.

Ferrero, L. 2009. Foraminíferos y ostrácodos del Pleistoceno tardío (Mar Chiquita, provincia de Buenos Aires, Argentina). Ameghiniana, 46:637-656.

Ganning, B. 1971. On the ecology of Heterocypris salinus, $H$. incongruens and Cypridopsis aculeata (Crustacea: Ostracoda) from Baltic brackish-water rockpools. Marine Biology, 8:271279. doi:10.1007/BF00348009

Gibson, T.G. \& Buzas, M.A. 1973. Species diversity: patterns in modern and Miocene Foraminifera of the eastern margin of North America. Geological Society of America Bulletin, 84:217-238. doi:10.1130/0016-7606(1973)84<217:SDPIMA>2.0.CO;2

Gómez, E.A.; Martínez, D.E.; Borel, C.M.; Guerstein, G.R. \& Cusminsky, G.C. 2005. Submarine evidence of Holocene sealevel fluctuations in the Bahía Blanca Estuary, Argentina. Journal of South American Earth Sciences, 20:139-155. doi:10.1016/j.jsames.2005.06.011

Karanovic, I. 2012. Recent freshwater ostracods of the world. Crustacea, Ostracoda, Podocopida. Heidelberg, Springer, 608 p. doi:10.1007/978-3-642-21810-1

Kidwell, S.M. \& Bosence, D.W.J. 1991. Taphonomy and timeaveraging of marine shelly faunas. In: P.A. Allison \& D.E.G. Briggs (eds.) Taphonomy: releasing the data locked in fossil record, New York, Plenum Press, p. 115-209 (Topics in Geobiology 9).

Kihn, R.G.; Cusminsky, G.C.; Mehl, A. \& Zárate, M. 2017 b. Asociaciones de ostrácodos de ambientes fluviales áridos, centro 
sur de la provincia de Mendoza, Argentina. In: CONGRESO DE CONSERVACIÓN DE LA BIODIVERSIDAD, 4, 2017. Libro de resúmenes, Río Negro, p. 69.

Kihn, R.G.; Martínez, D.E. \& Gómez, E.A. 2017a. Asociaciones de ostrácodos del intermareal del estuario de Bahía Blanca, Argentina. Revista Brasileira de Paleontologia, 20:90-100. doi:10.4072/rbp.2017.1.07

Kihn, R.G.; Martínez, D.E.; Gómez, E.A. \& Borel, C.M. 2016. Asociaciones de ostrácodos bentónicos actuales y del Holoceno del estuario de Bahía Blanca (Buenos Aires, Argentina): interpretaciones paleoambientales. Revista Brasileira de Paleontologia, 19:465-480. doi:10.4072/rbp.2016.3.11

Kontrovitz, M. 1975. A study of the differential transportation of ostracodes. Journal of Paleontology, 49:937-941.

Kotzian, S.C.B. 1974. New fresh-water ostracodes of the genus Chlamydotheca from Brazil. Ecology, geographic distribution and stratigraphical position. Anais da Academia Brasileira de Ciencias, 46:423-467.

Laprida, C. 2006. Ostrácodos recientes de la llanura pampeana, Buenos Aires, Argentina: ecología e implicancias paleolimnológicas. Ameghiniana, 43:181-204.

Martens, K. \& Behen, F. 1994. A checklist of the Recent non-marine ostracods (Crustacea, Ostracoda) from the Inland waters of South America and adjacent islands. Luxembourg, Musée National d'Historie Naturelle de Luxembourg, 84 p. (Travaux Scientifiques 22).

Martens, K. \& Savatenalinton, S. 2011. A subjective checklist of the Recent, free-living, non-marine Ostracoda (Crustacea). Zootaxa, 2855:1-79.

Martín-Rubio, M.; Elorza-Remón, M.; Rodriguez-Lazaro, J. \& Pascual, A. 2006. Distribución areal y ecología de las asociaciones de ostrácodos recientes en la marisma Joyel (Cantabria). Geogaceta, 40:187-190.

Martínez, D.E. 2002. Microfauna (Ostracoda, Crustacea) del Cuaternario de las cercanías de Bahía Blanca, República Argentina. Universidad Nacional del Sur, Tesis doctoral, 378 p.

Martínez, D.E. 2005. Asociaciones de ostrácodos modernos del estuario de Bahía Blanca, Buenos Aires, Argentina. Ameghiniana, 42:669-684.

Meisch, C. 2000. Freshwater Ostracoda from western and central Europe. Heidelberg, Spektrum Akademischer Verlag, 523 p.

Mezquita, F.; Roca, J.R.; Reed, J.M. \& Wansard, G. 2005. Quantifying species-environment relationships in non-marine Ostracoda: examples using Iberian data. Palaeogeography, Palaeoclimatology, Palaeoecology, 225:93-117. doi:10.1016/j. palaeo.2004.02.052

Mezquita, F.; Tapia, G. \& Roca, J.R. 1999. Ostracoda from springs on the eastern Iberian Peninsula: ecology, biogeography and palaeolimnological implications. Palaeogeography, Palaeoclimatology, Palaeoecology, 148:65-89. doi:10.1016/ S0031-0182(98)00176-X

Moguilevsky, A. \& Whatley, R. 1995. Crustacea Ostracoda. In: E. Lopretto \& G. Tell (eds.) Ecosistemas de aguas continentales. Metodologías para su estudio, Eudeba, p. 973-999.

Moore, R. \& Pitrat, C.W. 1961. Treatise on Invertebrate Paleontology. Part Q Arthropoda 3. Crustacea, Ostracoda. Lawrence, Geological Society of America and University of Kansas Press, 442 p.

Müller, O.F. 1776. Zoologiae Danicae Prodromus: seu Animalium Daniae et Norvegiae indigenarum; characteres, nomina, et synonyma imprimis popularium. Havniae, $282 \mathrm{p}$.

Murray, J.W. 1973. Distribution and ecology of living benthic foraminiferids. London, Heinemann Educational Books, 274 p.
Pearson, T.H. \& Rosenberg, R.1978. Macrobenthic succession in relation to organic enrichment and pollution of the marine environment. Oceanography and Marine Biology Annual Review, 16:229-311.

Pinto, I.D. \& Kotzian, S.C.B. 1961. Novos ostracodes da Familia Darwinulidae e a variação das impressões musculares. Porto Alegre, Instituto de Ciências Naturais da Universidade do Rio Grande do Sul, p. 5-64 (Boletim 6).

Purper, I. \& Würdig, N.L. 1974. Occurrence of Heterocypris incongruens (Ramdohr), 1808 - Ostracoda - in Rio Grande do Sul, Brazil. Discussion on the allied genera: Cyprinotus, Hemicypris, Homocypris and Eucypris. Pesquisas, 3:69-91.

Rahmdor, F.A. 1808. Übber die gattung Cypris Müller und drei zur derselben gehorige neue Arten. Gesellschaft Naturforschender Freunde zu Berlin, 2:83-91.

Ramírez, F.C. 1967. Ostrácodos de lagunas de la provincia de Buenos Aires. Revista del Museo de La Plata, Nueva Serie Zoología, 73:5-79.

Ramón-Mercau, J.; Plastani, M.S. \& Laprida, C. 2014. A review of the genus Limnocythere (Podocopida: Limnocytheridae) in the Pampean region (Argentina), with the description of a new species, Limnocythere cusminskyae sp. nov. Zootaxa, 3821:26-36. doi:10.11646/zootaxa.3821.1.2

Reichholf, V.J. 1983. Ökologie und Verhalten des Muschelkrebses Heterocypris incongruens Claus, 1892 (Ostracoda). Spixiana, 6:205-210

Schäfer, H.W. 1943. Über zwei neue deutsche Arten der SüsswasserOstracoden. Zoologischer Anzeiger, 143:210-216.

Scheffer, M.; Carpenter, S.; Foley, J.A.; Folke, C. \& Walker, B. 2001b. Catastrophic shifts in ecosystems. Nature, 413:591-596. doi:10.1038/35098000

Scheffer, M.; Straile, D.; Van Nes, E.H. \& Hosper, H. 2001a. Climatic warming causes regime shifts in lake food webs. Limnology and Oceanography, 46:1780-1783. doi:10.4319/lo.2001.46.7.1780

Schwalb, A.; Burns, S.J.; Cusminsky, G.; Kelts, K. \& Margraff, V. 2002. Assemblage diversity and isotopic signals of modern ostracodes and host waters from Patagonia, Argentina. Palaeogeography, Palaeoclimatology, Palaeoecology, 187:323339. doi:10.1016/S0031-0182(02)00484-4

Staff, G.M.; Stanton, R.J.; Powell, E.N. \& Cummins, H. 1986. Timeaveraging, taphonomic and their impact on paleocommunity reconstruction: death assemblages in Texas bays. Geological Society of America Bulletin, 97:428-443. doi:10.1130/00167606(1986)97<428:TTATIO>2.0.CO;2

Whatley, R.C. 1988a. Ostracoda and palaeogeography. In: P. Decker; J.P. Colin \& J.P. Peypouquet (eds.) Ostracoda in the Earth Sciences, Elsevier, p. 103-123.

Whatley, R.C. 1988b. Population structure of ostracods: some general principles for the recognition of palaeoenvironments. In: P. Deckker; J.P. Colin \& J.P. Peypouquet (eds.) Ostracoda in the Earth Sciences, Elsevier, p. 245-256.

Wierzejski, A. 1893. Skoripuaki i wrotki (Rotatoria) slodkowodne zebrane W Argentynie. Wydzial Matematyczno-Przyrodniczy, 24:229-249.

Zabert, L. 1981. Ostrácodos cuaternarios de Taco Pozo (provincia de Chaco, Argentina) con algunas consideraciones paleoecológicas. Facena, 4:77-87.

Zabert, L. \& Herbst, R. 1986. Ostrácodos Pleistocénicos del arroyo Perucho Verna, provincia de Entre Ríos, Argentina. Ameghiniana, 23:213-224.

Received in May, 2017; accepted in November, 2017. 